\title{
Consensus on Virtual Management of Vestibular Disorders: Urgent Versus Expedited Care
}

\author{
Aasef G. Shaikh ${ }^{1}$ (D) - Adolfo Bronstein ${ }^{2} \cdot$ Sergio Carmona ${ }^{3} \cdot$ Yoon-Hee Cha ${ }^{4} \cdot$ Catherine Cho $^{5} \cdot$ Fatema F. Ghasia $^{6}$. \\ Daniel Gold ${ }^{7} \cdot$ Kemar E. Green $^{7} \cdot$ Christoph Helmchen $^{8} \cdot$ Richard T. Ibitoye $^{2} \cdot$ Jorge Kattah $^{9} \cdot$ Ji-Soo Kim ${ }^{10}$.

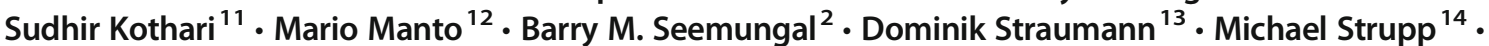 \\ David Szmulewicz $^{15}$ - Alexander Tarnutzer ${ }^{13,16}$. Ali Tehrani ${ }^{7}$ Caroline Tilikete $^{17} \cdot$ Miriam Welgampola $^{18}$. $^{18}$ \\ ${\text { Guillermo } \text { Zalazar }^{3} \text { - Amir Kheradmand }}^{7}$
}

Published online: 14 August 2020

(C) This is a U.S. Government work and not under copyright protection in the US; foreign copyright protection may apply 2020

\begin{abstract}
The virtual practice has made major advances in the way that we care for patients in the modern era. The culture of virtual practice, consulting, and telemedicine, which had started several years ago, took an accelerated leap as humankind was challenged by the novel coronavirus pandemic (COVID19). The social distancing measures and lockdowns imposed in many countries left medical care providers with limited options in evaluating ambulatory patients, pushing the rapid transition to assessments via virtual platforms. In this novel arena of medical practice, which may form new norms beyond the current pandemic crisis, we found it critical to define guidelines on the recommended practice in neurotology, including remote methods in examining the vestibular and eye movement function. The proposed remote examination methods aim to reliably diagnose acute and subacute diseases of the inner-ear, brainstem, and the cerebellum. A key aim was to triage patients into those requiring urgent emergency room assessment versus non-urgent but expedited outpatient management. Physicians who had expertise in managing patients with vestibular disorders were invited to participate in the taskforce. The focus was on two topics: (1) an adequate eye movement and vestibular examination strategy using virtual platforms and (2) a decision pathway providing guidance about which patient should seek urgent medical care and which patient should have non-urgent but expedited outpatient management.
\end{abstract}

Electronic supplementary material The online version of this article (https://doi.org/10.1007/s12311-020-01178-8) contains supplementary material, which is available to authorized users.

Aasef G. Shaikh

aasefshaikh@gmail.com

1 Department of Neurology, University Hospitals, Cleveland VA Medical Center, 11100 Euclid Avenue, Cleveland, OH 44110, USA

2 Division of Brain Sciences, Imperial College, London, UK

3 INEBA Institute of Neurosciences Buenos Aires, Buenos Aires, Argentina

4 Department of Neurology, University Minnesota, Minneapolis, MN, USA

5 Departments of Neurology and Otolaryngology, NYU Grossman School of Medicine, New York, NY, USA

6 Cole Eye Institute, Cleveland Clinic, Cleveland, OH, USA

7 Department of Neurology, Johns Hopkins University, Baltimore, MD, USA

8 Department of Neurology, University of Lübeck, Lübeck, Germany

9 Department of Neurology, University of Illinois, Peoria, IL, USA
10 Department of Neurology, Seoul National University, Seoul National University Bundang Hospital, Seongnam, South Korea

11 Department of Neurology, Pune Hospital, Pune, India

12 Department of Neurology, CHU-Charleroi, University of Mons, Mons, Belgium

13 Department of Neurology, University Hospital, Zurich, Switzerland

14 Department of Neurology, Ludwig-Maximilians University, Munich, Germany

15 Balance Disorders \& Ataxia Service, The Royal Victorian Eye \& Ear Hospital, Victoria, Australia

16 Department of Neurology, Cantonal Hospital of Baden, Baden, Switzerland

17 Department of Neurology, Hospices Civils de Lyon, Lyon, France

18 Department of Neurology, Central Clinical School, University of Sydney, Sydney, Australia 
Keywords Cerebellum $\cdot$ Brainstem $\cdot$ Emergency room $\cdot$ Stroke $\cdot$ Vestibular neuritis

\section{The Challenge}

Disorders that rapidly $(<72 \mathrm{~h})$ lead to severe vertigo, postural imbalance, intolerance to head movements, nausea/vomiting, with or without nystagmus, define the acute vestibular syndrome (AVS) [1-3]. The AVS results in about 2.6 million visits to the US emergency rooms each year [3]. The central causes of AVS such as stroke or inflammatory disease (e.g., multiple sclerosis) are common and they require immediate medical attention [4-6]. Under the crisis in a pandemic and especially when the world is eager to resume activities, physicians will be challenged in triaging patients based on priorities: which patient has to be attended urgently and who needs to be seen expeditiously as an outpatient? The crisis further complicates the challenge related to the pre-existing scarcity of healthcare providers with expertise in medical neurotology. Hence, there is a pressing need for a globally accepted strategy that can assist providers who are not specialized in neurotology to decide which dizzy patient needs to be directed to the emergency department urgently versus who can be seen as an outpatient expeditiously and how to implement remote assessments using virtual platforms to make such critical decisions.

\section{The Solution}

We invited a taskforce of vestibular and eye movement experts who have substantial clinical experience. This taskforce supported by the Society for Research in Cerebellum and Ataxia addressed strategic approaches on (1) implementing a virtual platform for remote assessment of the dizzy patient and (2) triaging strategy, i.e., who to advise to go to the emergency room for urgent medical attention versus who should be seen expeditiously in the outpatient clinic. We based our guidelines on the taskforce members' practical experience. It was obvious that a focused history invariably identified diplopia, acute unilateral deafness, and other brainstem or cerebellar deficits including sensory deficits, focal weakness, or inability to maintain posture $[2,7]$. These deficits often suggest central etiology requiring urgent attention. We then consolidated the taskforce members' practice patterns to prepare suggestions for virtual assessment and triaging strategies.

\section{Strategies for the Virtual Examination of Eye Movements and Vestibular System}

The proposed strategies for the virtual examination of eye movements and the vestibular system did not require specialized equipment. The taskforce members found these strategies effective in triaging patients and reaching an accurate diagnosis. Two forms of practices were observed: synchronous and asynchronous. The synchronous virtual examination, that is live videoconference between the patient and the provider using two-way audiovisual link, was more common among our taskforce members compared with the asynchronous virtual examination when the patient captured a video of his/her deficit(s) and transferred it to the provider using a secure link. The advantage of the synchronous virtual examination is that it allows direct and real-time interaction with the patients. The advantage of an asynchronous examination is that the patients are able to capture paroxysmal events with usually better video resolution or perform video recordings at their convenience.

\section{Synchronous Virtual Examination}

Videoconferencing using a two-way audiovisual link is the key aspect of the synchronous virtual examination. The taskforce members used different virtual platforms including commercially available programs such as Zoom, Doxy.Me, Cisco Jabber, WhatsApp, WebEx, Skype, and FaceTime. The taskforce does not endorse any specific platform or provide assurance that they are secure. There are usually legal restrictions placed on the use of some platforms by a provider's institution or government. Nevertheless, we point out that some virtual platforms are specifically dedicated to healthcare. Although patients generally find them easy to use, sometimes, guidance over phone or assistance from more technologically experienced family members was required.

Besides face-to-face conversations and history taking, the virtual platform allowed basic elements of the examination including mental state, speech, head tilt, examination of eye movements, facial palsy, muscle power (if a caregiver could assist or by checking for asymmetry while keeping both arms up with the eyes closed), coordination (ataxia or hyperkinetic movement disorders), and gait (if a caregiver was also present), to be performed. The taskforce suggested the following pragmatic approach using simple clinical tests.

General Observation The general appearance of the patient could yield important diagnostic information. An abnormal tilt of the head when sitting suggests an ocular tilt reaction reflecting an imbalance in the otolith pathway due to acute etiologies such as stroke or exacerbation of multiple sclerosis.

Binocular Eye Position A detailed history of diplopia including its onset, constant versus intermittent, binocular versus 
monocular, horizontal versus vertical, at distance or at near, and its dependence on the gaze direction is critical to diagnose the origin. By looking at the patient's gaze straight ahead into the camera, vertical and/or horizontal binocular misalignment can be noticed. Comparing the image of the cellphone or computer monitor on the two pupils can determine the misalignment in the two eyes. Reflection of the computer or cell phone screen can be used to assess the symmetry of light reflexes. One eye can be occluded with a large opaque spoon or the patient's hand. Incomitant strabismus can be detected by a cover-uncover test in a patient is seated away from the video camera. Pupil and lid asymmetries can be observed by asking the patient to move close to the camera.

Nystagmus It is possible to observe spontaneous nystagmus on virtual platforms. Sometimes, it is important for the patient to get closer to the camera with fully illuminated eyes. Virtual examination of nystagmus in supine or ear-down orientation either requires the use of the cellular phone as a patient interface or detachable desktop camera. If the patient is unable to maneuver the camera in supine or ear-down orientation, then it would be ideal to get help from the caregiver.

Saccades It is possible to examine saccade metrics (i.e., slowing and dysmetria) by alternating quick gaze shifts with the head stationary from the right (lower) to the left (upper) corner rim of the monitor. In the case of a smartphone, other targets in the patient's room need to be used.

Pursuit This can be examined with the phone or web camera in the patient's hand. Using the camera as the target, the patient moves the camera slowly in an arc toward the left, right, up, and down with the head relatively stationary while carefully fixating on the camera. Pursuit can also be examined by asking the patient to follow slowly moving finger of the examiner.

Convergence This can be examined as the patient moves the camera slowly towards the eyes while looking at the camera. The alternate way of examining the convergence is to look at the patient's own finger moving towards or away from the eyes.

Vestibulo-Ocular Reflex and Its Suppression The visual vestibulo-ocular reflex can be examined as the patient moves the head while the eyes fixate on the stationary camera. The cancelation of the vestibulo-ocular reflex can be seen when the patient rotates the body to the left and right while holding the camera with the hand. The latter requires a smartphone or a detachable web camera. The head impulse test can be more challenging to elicit on audiovisual platforms; however, the members of the taskforce considered an alternate strategy of instructing the patient to make active rapid head rotations while keeping the gaze fixated on the stationary camera. Albeit the taskforce appreciates the differences in the neurobiology of corrective saccade generation from active versus passive head movements (i.e., with a better vestibuloocular reflex performance during active head movements increasing the risk of false-negative head impulse test), the active head impulse was the most feasible way to implement the head impulse test in virtual clinical examination.

Stance and Gait Romberg and Fukuda testing can be performed while standing in the corner of the room (for safety) with the camera kept on the floor or propped against support to capture the entire body or video filmed by a caregiver. Gait can be assessed in the same way with the camera on the floor while the patient walks towards and away from it.

Hearing Deafness in conjunction with eye movement and vestibular deficits provides an important diagnostic element. Hearing can be tested using a ticking watch (high frequency) or finger taps (lower frequency) or by swapping ears on the phone to screen for subjective changes in the hearing. A reasonable hearing assessment can be done with Internet-based systems, e.g., https://hearingtest.online. Although online hearing assessments may have general problems such as calibrating the headphones/device in the presence of the ambient noise, they are good enough to establish significant hearing asymmetry.

\section{Asynchronous Virtual Examination}

The taskforce also recognized that the video frame rate (i.e., the number of frames per second, fps) can impact the detection of abnormal eye movements. In general, video frame rates below 60 fps may not be enough to reliably capture nystagmus with small excursions and frequent quick phases, ocular flutter, or subtle changes in saccades. Suboptimal video quality is possible as webbased audiovisual platforms are vulnerable to slowing of network speed, the inability of patients to maneuver the camera, or poor lighting or camera resolution. An asynchronous virtual examination can address this limitation. The patients' eye movements can be recorded with their video recording devices and transferred using a secure web-based platform to the provider. Such higherresolution videos can help the physician to detect finer, higher frequency, and smaller amplitude eye movements, as well as subtle changes in saccade velocities or abnormalities in the amplitude or direction of the vestibuloocular reflex. Asynchronous examinations will allow the ability to find appropriate lighting and additional help from the patient's caretaker. Hand-held video cameras, as built into the smartphones, allow eye movements to be recorded at different body and head orientations, which could be instrumental for the diagnosis of positional nystagmus or effectively performing Dix-Hallpike or Sémont maneuvers at home by seeing their own eyes clearly with 
the video camera in "selfie" mode. Patients, however, need instructions on how to capture eye movements successfully.

Table 1 summarizes the techniques recognized by the taskforce for examining eye movements and vestibular function using virtual platforms. The video graphic demonstration of comprehensive eye movement and vestibular examination in a healthy volunteer is illustrated in Supplementary Material 1. Additional video links in the Supplementary Material 1 depict an example of a synchronous video recording showing saccade palsy and an asynchronous video recording showing hypoactive vestibulo-ocular reflex. Supplementary Material 2 depicts synchronous examination depicting gaze-evoked jerk nystagmus and curved trajectory of vertical saccade in a patient with the atrophy of cerebellar vermis.

\section{Determining the Level of In-Person Care}

It is vital to know which dizzy patient should seek immediate care in the emergency department and which patient should receive non-emergent but expedited care. The taskforce provided the following insight on this important challenge.

\section{Urgent Admission to the Emergency Department}

Historical features in favor of immediate admission to the emergency department include the first spell of acute imbalance, advanced age, and vascular risk factors. The following clinical signs also suggest a need for urgent evaluation.

1. Concomitant signs of the brainstem and/or cerebellar dysfunction such as speech problems, diplopia, unilateral limb weakness, ataxia, clumsiness, paresthesia, or numbness, and hearing loss.

2. It is also important to determine the extent of disability caused by symptoms, whether the patients can walk, and how rapidly the symptoms have developed. It is possible to use virtual platforms to classify truncal ataxia according to their severity levels: grade 1 as mild to moderate imbalance but is able to walk independently; grade 2 as severe imbalance with standing but cannot walk without support; and grade 3 as falling in an upright posture. The likelihood of stroke is very high in the case of grades 2 and 3 truncal ataxia; when associated with nystagmus of central origin, it is almost always an acute stroke [2].

3. Headaches, particularly in the occipital or sub-occipital region, can frequently occur in those with posterior fossa
Table 1 Techniques recognized by the taskforce for examining eye movements and vestibular function using virtual platforms

Exam $\begin{aligned} & \text { Description } \\ & \text { Nystagmus } \\ & \text { 1. Instruct the patient to move close to the camera with eyes fully illuminated. } \\ & \text { 2. Ask the patient to stare straight ahead at the camera, then assess for nystagmus or } \\ & \text { other abnormalities. } \\ & \text { 3. Ask the patient to look approximately } 15 \text { deg rightward, leftward, upward, or } \\ & \text { downward, holding each position for at least } 10 \mathrm{~s} \text { (for down and down/lateral gazes } \\ & \text { instruct patient/family member to elevate eyelids to ensure visualization of the eyes). } \\ & \text { 4. Evaluate nystagmus under closed eyelids by looking at the corneal bulges or instruct } \\ & \text { caregiver/patient to perform a modified penlight-cover test using a cellphone flash- } \\ & \text { light to examine fixation-removed nystagmus } \\ & \text { 1. Instruct the patient to look straight at the camera. } \\ & \text { 2. Ask the patient to shift gaze to an eccentric location, such as the rim of the computer } \\ & \text { monitor or objects on the right or the left of the room at the examiner's instruction. } \\ & \text { 3. Ask the patient to shift gaze up or down at the examiner's instruction. } \\ & \text { 1. Instruct the patient to look at the camera. } \\ & \text { 2. Ask the patient to move the camera smoothly to the right or to the left while fixating } \\ & \text { on the camera. } \\ & \text { 1. Instruct the patient to move close to the camera with eyes fully illuminated. } \\ & \text { 2. Look for asymmetry in the corneal light reflection. } \\ & \text { 3. Ask the patient to use their palm to cover and uncover one eye. The examiner watches } \\ & \text { the movement of the eye after it is uncovered. } \\ & \text { 4. Ask the patient to perform alternating monocular occlusion using the palm of the } \\ & \text { hand for 2-3 s at a time and/or following the physician's direction. } \\ & \text { 1. Instruct the patient to move close to the camera with eyes fully illuminated } \\ & \text { 2. Ask the patient to fixate on a shared visual target in the center of the screen and } \\ & \text { rapidly move the head in the horizontal plane at the direction of the physician. }\end{aligned}$
alignment


strokes. Although a patient with recurrent episodes of headaches and vertigo is more likely to have vestibular migraine [8], the first episode could be a brainstem/ cerebellar stroke and should be referred to the emergency department.

4. The taskforce recommends that those patients with AVS putatively due to exacerbation of inflammatory reaction such as multiple sclerosis should be referred immediately to the emergency department for further evaluation and treatment.

5. Certain patients with AVS due to vestibular neuritis who do not fit typical characteristics or those with positional vertigo, who do not fit typical characteristics of benign paroxysmal positional vertigo (BPPV) $[9,10]$, should be referred to the emergency department.

\section{Non-urgent But Expedited Outpatient Investigation}

The taskforce members recommended a lower threshold for further investigation when subacute symptoms are progressing, lateralized, or if during examination there are clear central signs such as gaze-evoked nystagmus or saccade dysmetria. It is also possible that common acute disorders such as strokes can be present in the subacute phase. The taskforce offers the following guidelines for identifying conditions that require non-urgent but expedited outpatient evaluation and management.

1. Progressive deficits of eye movement and vestibular function or truncal and gait ataxia may indicate posterior fossa space-occupying lesions or subacute stroke.

2. The patients with severe forms of BPPV that do not follow a focused questionnaire [9] and positional testing performed during a virtual exam or do not resolve with virtually directed exercises should also be directed for an expedited in-person evaluation.

3. The patients with recurrent "spells" of vertigo, without any inter-ictal deficits may have compromise in the posterior circulation and is under risk for stroke. Prompt evaluation in the form of imaging and management for putative vascular etiologies is warranted in these patients.

The proposed guidelines were found effective during the clinical practice of the taskforce members. However, we emphasize that if there is any doubt, unusual features, or lack of clarity in the acute or subacute presentation of a novel symptom, or inability to accurately examine the patient due to technical limitations, the providers should have a low threshold to direct the patient to the emergency department for expedited clinical evaluation. It is also not uncommon that patients are not willing to visit the emergency room leading to inpatient ward admission, either due to fear of pandemics or due to financial reasons. In such situations, it may be possible for the physician to arrange for an immediate MRI at an outpatient facility possibly getting the result the same day.

We also emphasize that the goal of this paper is to propose the virtual practice patterns agreed upon by the taskforce members. The current paper does not provide data on how these patterns are effective objectively. Future investigations are required to apply these practice patterns and objectively measure the outcomes.

The application of telemedicine will resolve many logistical and financial challenges that are inherent to patients who suffer from neurological disorders. This could mean a major paradigm shift in healthcare practice. A word of caution however is required. The human interaction during in-person consultation will have to continue as an important component in the healing process and future guidelines for telemedicine should weigh the pros and cons of in-person versus telemedicine consultations.

\section{References}

1. Hotson JR, Baloh RW. Acute vestibular syndrome. N Engl J Med. 1998;339(10):680-5.

2. Carmona $\mathrm{S}$, et al. The diagnostic accuracy of truncal ataxia and HINTS as cardinal signs for acute vestibular syndrome. Front Neurol. 2016;7:125.

3. Kattah JC, Talkad AV, Wang DZ, Hsieh YH, Newman-Toker DE. HINTS to diagnose stroke in the acute vestibular syndrome: threestep bedside oculomotor examination more sensitive than early MRI diffusion-weighted imaging. Stroke. 2009;40(11):3504-10.

4. Tarnutzer AA, Berkowitz AL, Robinson KA, Hsieh YH, NewmanToker DE. Does my dizzy patient have a stroke? A systematic review of bedside diagnosis in acute vestibular syndrome. CMAJ. 2011;183(9):E571-92.

5. Pula JH, Newman-Toker DE, Kattah JC. Multiple sclerosis as a cause of the acute vestibular syndrome. J Neurol. 2013;260(6): 1649-54.

6. Newman-Toker DE. Missed stroke in acute vertigo and dizziness: it is time for action, not debate. Ann Neurol. 2016;79(1):27-31.

7. Seemungal B, Bronstein AM. A practical approach to acute vertigo. Pract Neurol. 2008;8(4):211-21.

8. Lempert T, Olesen J, Furman J, Waterston J, Seemungal B, Carey J, et al. Vestibular migraine: diagnostic criteria. J Vestib Res. 2012;22(4):167-72.

9. Kim HJ, Song JM, Zhong L, Yang X, Kim JS. Questionnaire-based diagnosis of benign paroxysmal positional vertigo. Neurology. 2020;94(9):e942-9.

10. von Brevern M, Bertholon P, Brandt T, Fife T, Imai T, Nuti D, et al. Benign paroxysmal positional vertigo: diagnostic criteria. J Vestib Res. 2015;25(3-4):105-17.

Publisher's Note Springer Nature remains neutral with regard to jurisdictional claims in published maps and institutional affiliations. 